\title{
Please please me
}

SW Choi *, PhD, David MH Lam, MB, ChB

\author{
Department of Anaesthesiology, The University of Hong Kong, Queen Mary Hospital, Pokfulam, Hong Kong
}

*Corresponding author: htswchoi@hku.hk

Hong Kong Med J 2016;22:85-6

DOI: 10.12809/hkmj154738

The placebo effect is getting stronger. The pharmaceutical industry spends billions of dollars on drug development each year, ${ }^{1,2}$ culminating in phase III trials whose aim is to prove the superior effectiveness of a new drug to a placebo. Many phase III trials have been discontinued, however, because the test drug was no better than placebo, at an estimated loss of half a billion US dollars. But if the placebo effect is all in the mind, ${ }^{3,4}$ how can it be getting stronger?

The very existence of a placebo effect is a point of contention for many scientists, with some vehemently stating that there is no such thing, and others claiming to have identified a "placebo gene. ${ }^{5}$ From the Latin for "I will please", the placebo effect is defined as any health effect measured after an intervention that is something other than a physiological response to a biologically active treatment. Although the 'placebo effect' has long been recognised, scientists only began to pay attention to it after the call for standardisation of clinical trials by the Cornell Conferences on Therapy in $1946 .{ }^{6}$ Factors that contribute to the measured placebo effect will differ depending on the situation, and a larger placebo effect may be seen with a subjective outcome, for example, pain, general wellbeing, and depression scores. ${ }^{3}$ When the outcome is more physiological, the placebo effect becomes smaller (or disappears), for example, in cancer or infectious disease trials.

Opponents to the existence of a placebo effect argue that its effects as measured in clinical trials are a combination of several other factors, including natural healing, where pathological conditions heal spontaneously, and regression to the mean, where the inclusion of patients with very high or very low values at the start of a study gives the illusion that statistical variation in later measurements is due to the effect of the treatment. ${ }^{7}$ Other possible explanations include the Hawthorne effect, when participants in a study change their behaviour, simply because they are in a study, or report better outcomes to please the clinicians/researchers conducting the study. ${ }^{8}$ Opponents believe that the placebo effect has been observed to be 'stronger' in recent clinical trials because trials are now better conducted, with true randomisation and participant/investigator blinding. Or it could be that drugs involved in discontinued phase III trials were simply not that effective.

Proponents of the placebo argue that not only is it effective for various conditions, from pain and depression to irritable bowel syndrome, but the placebo can be administered without deception. In other words, the sugar pill can work, even when patients know they have been prescribed a placebo. ${ }^{9}$ Supporters argue that because the placebo effect is so potent, treatments such as homeopathy, which has been shown countless of times to be no better than placebo in randomised controlled trials, should not be discontinued because it is effective, even if the treatment does nothing more than elicit a placebo response. A recent paper published in Science reviews the genetic basis of the placebo effect. ${ }^{10}$

Although a small study (104 subjects divided into three treatment groups), the review outlined how Hall et $\mathrm{al}^{11}$ found a linear relationship between polymorphisms in the gene encoding for catechol-Omethyltransferase (COMT) and the placebo response. The COMT enzyme breaks down catecholamines, and a common polymorphism can dictate either a valine (val), or methionine (met) at the amino acid position 158. It has been well-established that the met/ met form of COMT is less active, leading to higher concentrations of dopamine, and individuals with the met/met form have been correlated to higher levels of performance in cognitive tests when compared with individuals with the val/val variant. Hall et al ${ }^{11}$ showed that individuals with the met/met variant responded well to placebo, those with val/val showed little placebo response, and those with heterozygous (val/met) variant showed an intermediate response. The placebo response has been linked to dopamine release, and since the met/met variant of COMT is 3 to 4 times less efficient at breaking down dopamine, it may result in higher dopamine in the system, leading to a more intense feeling of pain relief in Hall et al's study, regardless of whether the patient was assigned the active drug, or placebo.

Hall et al's finding ${ }^{11}$ led colleagues at the Beth Israel Deaconess Medical Centre to file a patent on the concept of screening for, and then excluding, participants based on their COMT polymorphism before enrolling them into a clinical trial. If researchers can identify responders to the placebo, and exclude them from trials, they would have to recruit fewer participants and would see a bigger 
effect between the treatment and placebo arms, effectively shrinking the financial resources required for the clinical trial.

Although some of us may question the ethical issues involved in pre-screening of trial participants in such a way, this recent patent application is only one of several filed for clinical trial designs that can minimise the placebo effect. Other methods include what is known in the industry as a placebo run-in, where everybody is given a placebo at the beginning, and those participants who 'get too much better' are subsequently dropped from the trial when the participants are later randomised to the different arms. These strategies have not only led us to question the wording used on the informed consent form but also the subsequent regulations regarding the marketing of drugs that have been trialled in this way. When data are presented to drug regulatory bodies, will the regulators only allow these drugs to be used in those individuals with proven inability to respond to placebo?

\section{References}

1. Siddiqui M, Rajkumar SV. The high cost of cancer drugs and what we can do about it. Mayo Clin Proc 2012;87:93543.
2. Kaitin KI. Deconstructing the drug development process: the new face of innovation. Clin Pharmacol Ther 2010;87:356-61.

3. Howland RH. Understanding the placebo effect. Part 1: placebo use in clinical trials. J Psychosoc Nurs Ment Health Serv 2008;46:17-20

4. Miller FG, Colloca L, Kaptchuk TJ. The placebo effect: illness and interpersonal healing. Perspect Biol Med 2009;52:518-39.

5. Hall KT, Loscalzo J, Kaptchuk TJ. Genetics and the placebo effect: the placebome. Trends Mol Med 2015;21:285-94.

6. Gold H, Barr DP, DuBols EF, Cattell M, Wheeler $\mathrm{CH}$, editors. Cornell Conferences on Therapy. Vol 1. Calif Med 1946;65:42.

7. Price DD, Finniss DG, Benedetti F. A comprehensive review of the placebo effect: recent advances and current thought. Ann Rev Psychol 2008;59:565-90.

8. Miller FG, Rosenstein DL. The nature and power of the placebo effect. J Clin Epidemiol 2006;59:331-5.

9. Kaptchuk TJ, Friedlander E, Kelley JM, et al. Placebos without deception: a randomized controlled trial in irritable bowel syndrome. PLoS One 2010;5:e15591.

10. Servick K. Outsmarting the placebo effect. Science 2014;345:1446-7.

11. Hall KT, Lembo AJ, Kirsch I, et al. Catechol-Omethyltransferase val158met polymorphism predicts placebo effect in irritable bowel syndrome. PLoS One 2012;7:e48135. 